\title{
Real estimates of mortality following COVID-19 infection; an oversimplification of a complex issue
}

\author{
Alireza Hamidian Jahromi ${ }^{*}$, Hilda Mahmoudi \\ ${ }^{1}$ Department of Plastic Surgery, University of Tennessee Health Science Center, Memphis, Tennessee, USA \\ ${ }^{2}$ Department of Medical Education, Nova Southeastern University School of Osteopathic Medicine, Miami, USA
}

\section{Correspondence to:}

Alireza Hamidian Jahromi, Email: Alirezahamidian@yahoo. com, ahamidia@uthsc.edu

Received: 15 June 2020 Accepted: 2 July 2020 ePublished: 6 July 2020

Keywords: Coronavirus, COVID19, Epidemy, Mortality

\begin{abstract}
Core tip
Some authors have claimed that the mortality rate estimations for coronavirus disease 2019 (COVID-19) which are based on the number of deaths relative to the number of confirmed cases of infection are not representative of the actual death rate. As a matter of fact, the current mortality rate estimates (specially for calculations performed during the exponential phase of the virus spread) are an underestimation of the potential mortality of the symptomatic COVID-19 patients. Here we discuss some pitfalls of Baud and colleague's innovative suggestion to re-estimate the COVID-19 mortality rate by dividing the number of deaths on a given time by the number of confirmed symptomatic COVID-19 patients at an earlier time (time from onset of symptom to death).
\end{abstract}

$\mathrm{R}$ ecently Baud and his colleagues claimed that the mortality rate estimations for coronavirus disease 2019 (COVID-19) which are based on the number of deaths relative to the number of confirmed cases of infection are not representative of the actual death rate. They stated that patients who die on any given day were infected much earlier, and therefore the denominator of the mortality rate should be the total number of patients infected at the same time as those who died.

We agree to some extent with Baud et al, that the current mortality rate estimates are an underestimation of the potential mortality of the symptomatic COVID-19 patients (1). As we have just reached the exponential phase of the COVID-19 pandemic, especially outside of China, we support the authors' innovative suggestion to re-estimate the COVID-19 mortality rate by dividing the number of deaths on a given time by the number of confirmed symptomatic COVID-19 patients at an earlier time (time from onset of symptom to death) however, in the same region (1). Baud mortality estimation is based on time elapsed between diagnostic tests, not the start of symptoms, and death which reflects availability of tests, delay in reporting, health policy and implemented criteria for testing the patients.

Variations in medical advice-seeking habits of different societies, political stance of some countries to underreport cases or deaths, different screening strategies even for symptomatic patients, diversity of diagnostic tests [RT-PCR (sensitivity $=63 \%$ for nasal swab, $32 \%$ for pharyngeal swab) (2), chestCT (sensitivity=97\%)(3), blood test [ELISA (sensitivity=93-99\%)] (4), potential paraanalytic/analytic pitfalls of COVID-19 tests (5), and the fact that at the peak of the epidemic some countries lack the resources to check (test) the symptomatic but less critical cases (those who potentially would not need respiratory support or hospital admission), makes real estimation of the COVID-19 mortality extremely challenging if not impossible. Some may argue that there is no point in performing diagnostic tests before placing the potential cases on quarantine specially in situations where clinical condition of the patient does not warrant hospital admission.

During their assessment, Baud et al overlooked a significant contributing factor when calculating the mortality rate; a growing number of critically ill cases piling up as the epidemy expands which significantly contributes to a higher mortality in the upcoming stages of epidemy. On the other hand, if the health-care system collapses during the peak of the spread, as happened in Italy and Spain, health-care providers have to choose between resuscitating the elderly 
or the young patients which could artificially increase the mortality rates even further.

Authors' contribution

$\mathrm{AH}$ and $\mathrm{HM}$ contributed to conception and design, literature search, writing and editing the manuscript.

Conflicts of interest

The authors declare no conflict of interest regarding the publication of this article.

Ethical considerations

Ethical issues (including plagiarism, data fabrication, double publication) have been completely observed by the authors.

\section{Funding/Support}

None.

\section{References}

1. Baud D, Qi X, Nielsen-Saines K, Musso D, Pomar L, Favre G.
Real estimates of mortality following COVID-19 infection. Lancet Infect Dis. 2020 Mar 12. pii: S1473-3099(20)30195-X. doi: 10.1016/S1473-3099(20)30195-X.

2. Wang W, Xu Y, Gao R, Lu R, Han K, Wu G, et al. Detection of SARS-CoV-2 in Different Types of Clinical Specimens. JAMA. 2020; doi: 10.1001/jama.2020.3786.

3. Ai T, Yang Z, Hou H, Zhan C, Chen C, Lv W, et al. Correlation of Chest CT and RT-PCR Testing in Coronavirus Disease 2019 (COVID-19) in China: A Report of 1014 Cases. Radiology. 2020. doi: 10.1148/radiol.2020200642.

4. Guo L, Ren L, Yang S, Xiao M, Chang D, Yang F, Dela Cruz CS, et al. Profiling Early Humoral Response to Diagnose Novel Coronavirus Disease (COVID-19). Clin Infect Dis. 2020. doi: 10.1093/cid/ciaa310.

5. Lippi G, Simundic AM, Plebani M. Potential preanalytical and analytical vulnerabilities in the laboratory diagnosis of coronavirus disease 2019 (COVID-19). Clin Chem Lab Med. 2020. doi: 10.1515/cclm-2020-0285. 